\title{
A Pest Management Epidemic Model with Time Delay and Stage-Structure
}

\author{
Yumin Ding ${ }^{1}$, Shujing Gao ${ }^{1}$, Yujiang Liu ${ }^{1}$, Yun $\operatorname{Lan}^{2}$ \\ ${ }^{1}$ Key Laboratory of Jiangxi Province for Numerical Simulation and Emulation Techniques, \\ Gannan Normal University, Ganzhou, China \\ ${ }^{2}$ Jiangxi Environmental Engineering Vocational College, Ganzhou, China \\ E-mail: gaosjmath@126.com \\ Received June 12, 2010; revised July 22, 2010; accepted July 25, 2010
}

\begin{abstract}
In this paper, an SI epidemic model with stage structure is investigated. In this model, impulsive biological control which release infected pest to the field at a fixed time periodically is considered, and obtained the sufficient conditions for the global attractivity of pest-extinction periodic solution and permanence of the system. We also prove that all solutions of the model are uniformly ultimately bounded. The sensitive analysis on the two thresholds and to the changes of the releasing amounts of infected pest is shown by numerical simulations. Our results provide a reliable tactic basis for the practice of pest management.
\end{abstract}

Keywords: Pest Management, Stage Structure, Impulsive System, Permanence

\section{Introduction}

Pests outbreak often cause serious ecological and economic problems, and the warfare between human and pests has sustained for thousands of years. With the development of society and the progress of science and technology, a great deal of pesticides were used to control pests, because they can quickly kill a significant portion of pest population and sometimes provide the only feasible method for preventing economic loss. However, pesticide pollution is also recognized as a major health hazard to human beings and beneficial insects. At present, more and more people are concerned about the effects of pesticide residues on human health and on the environment [1].

In natural world, there are many insects whose individual members have a life history that takes them through two stages, larva and mature. Pathogens may not be effective against laver, that is, the disease only attacks the susceptible mature pest population. For example, saltcedar leaf beetle is such a pest. Pest control strategies have been attracted many experts over the past years. Recently, stage-structured models have received much attraction [2,3]. However, the epidemic models with stage-structure have been seldom studied. Zhang et al. [4] introduced the pest based on the stage-structure model which incorporates a discrete delay and pulses in order to investigate how epidemics influence the pest control process. An SI model with impulsive perturbations on diseased pest and spraying pesticides at fixed moment is proposed and investigated in [5], which obtained the sufficient conditions of the global attractivity of pest-extinction periodic solution and permanence of the system.

Incidence plays a very important role in research of epidemic models, bilinear and standard incidence rates have been frequently used in classical epidemic models [6]. Several different incidence rates have been proposed by researchers. Anderson et al. pointed out that standard incidence is more suitable than bilinear incidence $[7,8]$. Levin et al. have adopted the incidence form like $\beta S^{q} I^{p}$ or $\beta S^{q} I^{p} / N$ [9]. Lindstrom pointed out the crowed incidence $\beta S(t) /\left(1+a S(t)+b S^{2}(t)\right)$ [10]. However there are seldom authors have concerned the stagestructured models under the simultaneous effect of disease and crowed incidence. A stage-structure model with the crowed incident rate is considered in [11]. According to the facts of pest management, we take the crowded effect as the incidence rate. Therefore, in this paper, a pest management epidemic model which with time delay and stage-structure is considered.

The present paper is organized as follows. In the next section, we formulate the pest management model. In Section 3, some essential lemmas which will be used to prove our main results are introduced. In Section 4, global attractivity of the susceptible pest-eradication pe- 
riodic solution and the permanence of the model is analyzed. In the final section, we present some numerical simulations to illustrate the results and point out some future research directions.

\section{Model Formulation}

In this paper, we study the pest management epidemic model:

$$
\left\{\begin{array}{l}
L^{\prime}(t)=B(S(t)) S(t)-\gamma L(t)-e^{-\gamma \tau} B(S(t-\tau)) S(t-\tau), \\
S^{\prime}(t)=e^{-\gamma \tau} B(S(t-\tau)) S(t-\tau)-\frac{\beta S(t) I(t)}{1+a S(t)+b S^{2}(t)}-\eta S(t), \\
I^{\prime}(t)=\frac{\beta S(t) I(t)}{1+a S(t)+b S^{2}(t)}-d I(t), \\
\Delta I(t)=I\left(t^{+}\right)-I(t)=\mu, \quad t=n T, \quad n \in Z_{+},
\end{array}\right\} t \neq n T,
$$

with initial conditions

$$
\left\{\begin{array}{l}
\left(\varphi_{1}(t), \varphi_{2}(t), \varphi_{3}(t)\right) \in C_{3}^{+} \text {for } t \in[-\tau, 0], \varphi_{i}(0)>0, i=1,2,3 \\
\varphi_{1}(0)=\int_{-\tau}^{0} e^{r \theta} B\left(\varphi_{2}(\theta)\right) \varphi_{2}(\theta) d \theta
\end{array}\right.
$$

where all the coefficients of model (1) are nonnegative and $L(t), S(t), I(t)$ represent the larva, mature susceptible and infected pest population at time $t$, respectively. The model is derived from the following assumptions.

$\left(\mathrm{H}_{1}\right)$ The death rate of larva population is proportional to the existing larva population with proportionality constant $\gamma$, the death rate of mature susceptible and infected pest population is proportional to the existing mature susceptible and infected pest population with proportionality constants $\eta$ and $d$, respectively.

$\left(\mathrm{H}_{2}\right)$ Only the susceptible pest population can reproduce. $B(S)$ is a birth rate function of the susceptible pest population for $S \in(0, \infty)$ with $B(S)$ is monotonically decreasing, $\lim _{S \rightarrow \infty} B(S)=B(\infty)$ exists and $B\left(0^{+}\right)>\eta>$
$\bar{\delta}>B(\infty)$, where $\bar{\delta}=\frac{1}{2} \min \{\eta, \gamma, d\}$.

$\left(\mathrm{H}_{3}\right) \tau$ represents a constant time to maturity, the product term $e^{-\gamma \tau} B(S(t-\tau)) S(t-\tau)$ describes that immature pest who were laid at time $t-\tau$ and survive at time $t$.

$\left(\mathrm{H}_{4}\right)$ The incident rate is the crowded effect.

$\frac{\beta S(t)}{1+a S(t)+b S^{2}(t)}$.

$\left(\mathrm{H}_{5}\right) \mu$ is the releasing amounts of infected pest at $t=n T, n=1,2, \cdots$, and $T$ is the period of the impulsive effect.

Before going into any detail, we simplify model (1) and restrict our attention to the following model:

$$
\left\{\begin{array}{l}
S^{\prime}(t)=e^{-\gamma \tau} B(S(t-\tau)) S(t-\tau)-\frac{\beta S(t) I(t)}{1+a S(t)+b S^{2}(t)}-\eta S(t), \\
I^{\prime}(t)=\frac{\beta S(t) I(t)}{1+a S(t)+b S^{2}(t)}-d I(t), \\
\Delta I(t)=\mu, \quad t=n T .
\end{array}\right\} t \neq n T,
$$

The initial conditions for (3) are

$$
\left(\varphi_{2}(t), \varphi_{3}(t)\right) \in C^{+}=C\left([-\tau, 0], R_{+}^{2}\right), \varphi_{i}(0)>0, i=2,3 .
$$

\section{Some Useful Lemmas}

The solution of system (1), denoted by $x(t)=(L(t)$, $S(t), I(t))^{T}$ is a piecewise continuous function $x: R_{+} \rightarrow R_{+}^{3}, \quad x(t)$ is continuous on $(n T,(n+1) T)$, $n \in Z_{+}$and $x\left(n T^{+}\right)=\lim x(t)$ exists. Before demonstrating the main results, we need to give some lemmas which will be used as follows.

Lemma 1. (see [11]). Let $\left(\varphi_{1}(t), \varphi_{2}(t), \varphi_{3}(t)\right)>0$ for $-\tau<t<0$. Then any solution of system (1) is strictly positive.

Lemma 2. Let the function $m \in P C^{\prime}\left[R^{+}, R\right]$ satisfies the inequalities

$$
\left\{\begin{array}{l}
m^{\prime}(t) \leq p(t) m(t)+q(t), t \geq t_{0}, t \neq t_{k}, k=1,2, \cdots, \\
m^{\prime}\left(t_{k}^{+}\right) \leq d_{k} m\left(t_{k}\right)+b_{k}, t=t_{k}
\end{array}\right.
$$

where $p, q \in P C\left[R^{+}, R\right]$ and $d_{k} \geq 0, b_{k}$ are constants. Then 


$$
\begin{aligned}
m(t) & \leq m\left(t_{0}\right) \prod_{t_{0}<t_{k}<t} d_{k} \exp \left(\int_{t_{0}}^{t} p(s) d s\right) \\
& +\sum_{t_{0}<t_{k}<t}\left(\prod_{t_{k}<t_{j}<t} d_{j} \exp \left(\int_{t_{0}}^{t} p(s) d s\right)\right) b_{k} \\
& +\int_{t_{0}}^{t} \prod_{s<t_{k}<t} d_{k} \exp \left(\int_{s}^{t} p(\sigma) d \sigma\right) q(s) d s, \quad t \geq t_{0} .
\end{aligned}
$$

The proof of this lemma is given in [12].

We now show that all solutions of (1) are uniformly ultimately bounded.

Lemma 3. Any solution $(L(t), S(t), I(t))$ of system (1) is uniformly ultimately bounded. That is, there exists a constant $M=\frac{\lambda}{\bar{\delta}}+\frac{\mu e^{\bar{\delta} T}}{e^{\bar{\delta} T}-1}>0$ such that $L(t) \leq M$, $\mathrm{S}(t) \leq M, \mathrm{I}(t) \leq M$ for sufficiently large $t$.

Proof. Define $V(t)=L(t)+S(t)+I(t)$. By simple computation when $t \neq n T$, we calculate the derivative of $V$ along the solution of system (1)

$D^{+} V(t)=B(S) S-\gamma L-\eta S-d I \leq B(S) S-2 \bar{\delta}(L+S+I)$, for $t \in(n T,(n+1) T)$.

Then we derive

$$
D^{+} V(t)+\bar{\delta} V \leq B(S) S-\bar{\delta} S, t \in(n T,(n+1) T) .
$$

Obviously, from conditions $\left(\mathrm{H}_{1}\right)$ and $\left(\mathrm{H}_{2}\right)$, we are easy to know that there exists a constant $\lambda>0$ such that

$$
D^{+} V(t)+\bar{\delta} V \leq \lambda, t \in(n T,(n+1) T),
$$

for $n$ large enough.

When $t=n T$, we get

$$
V\left(n T^{+}\right)=V(n T)+\mu .
$$

According to Lemma 2, we derive

$$
\begin{aligned}
V(t) & \leq V(0) e^{-\bar{\delta} t}+\int_{0}^{t} \lambda e^{-\bar{\delta}(t-s)} d s+\sum_{0<n T<t} \mu e^{-\bar{\delta}(t-n T)} \\
& \rightarrow \frac{\lambda}{\bar{\delta}}+\frac{\mu e^{\bar{\delta} T}}{e^{\bar{\delta} T}-1} \triangleq M \text { as } t \rightarrow \infty .
\end{aligned}
$$

Therefore by the definition of $V(t)$, we obtain that each positive solution of system (1) is uniformly ultimately bounded. This completes the proof.

Lemma 4. Consider the following delay differential equation:

$$
x^{\prime}(t)=a_{1} x(t-\tau)-a_{2} x(t)
$$

where $a_{1}, a_{2}$ and $\tau$ are all positive constants and $x(t)>0$ for $t \in[-\tau, 0]$. We have:

1) If $a_{1}<a_{2}$, then $\lim _{x \rightarrow \infty} x(t)=0$;

2) If $a_{1}>a_{2}$, then $\lim _{x \rightarrow \infty} x(t)=+\infty$.

The proof of this lemma is given in [13].
Lemma 5 (see [11]). Consider the following impulsive system:

$$
\begin{cases}v^{\prime}(t)=-d v(t), & t \neq n T, \\ v\left(n T^{+}\right)=v(n T)+\mu, & t=n T, \quad n=1,2, \cdots,\end{cases}
$$

where $d, \mu>0$. Then there exists a unique positive periodic of system (6)

$$
\tilde{v}(t)=v^{*} e^{-d(t-n T)}, \quad t \in(n T,(n+1) T], n \in Z_{+},
$$

which is globally asymptotically stable, where $v^{*}=\frac{\mu}{1-e^{-d T}}$.

\section{Main Results}

In this section that follows we determine the global attractivity condition of the susceptible pest-extinction periodic solution and the permanence of the system (3).

\subsection{Global Attractivity of the Susceptible Pest-Extinction Periodic Solution}

Denote

$$
R^{*} \triangleq \frac{\left(B(0) e^{-\gamma \tau}-\eta\right)\left(1+a M+b M^{2}\right)\left(e^{d T}-1\right)}{\mu \beta}
$$

where $M=\frac{\lambda}{\bar{\delta}}+\frac{\mu e^{\bar{\delta} T}}{e^{\bar{\delta} T}-1}$

Theorem 1. Let $(S(t), I(t))$ be any solution of system (3), the susceptible pest-extinction periodic solution $(0, \tilde{I}(t))$ of (3) is globally attractive provided that $R^{*}<1$.

Proof. Since $R^{*}<1$, we can choose $\varepsilon_{0}$ sufficiently small such that

$$
B(0) e^{-\gamma \tau}<\frac{\beta}{1+a M+b M^{2}}\left(\frac{\mu e^{-d T}}{1-e^{-d T}}-\varepsilon_{0}\right)+\eta
$$

Note that $I^{\prime}(t) \geq-d I(t)$, from Lemma 2 and Lemma 5 , we have that for the given $\varepsilon_{0}$ there exists an integer $k_{1}$ such that for $n T<t \leq(n+1) T, n>k_{1}$

$$
I(t)>\tilde{I}(t)-\varepsilon_{0} \geq\left(\frac{\mu e^{-d T}}{1-e^{-d T}}-\varepsilon_{0}\right) \triangleq \rho .
$$

From condition $\left(\mathrm{H}_{1}\right)$, (3) and (9), we yield

$$
\begin{aligned}
\frac{d S(t)}{d t} & \leq B(0) e^{-\gamma \tau} S(t-\tau) \\
& -\left(\frac{\beta}{1+a M+b M^{2}} \rho+\eta\right) S(t),
\end{aligned}
$$

for $t>n T+\tau, n>k_{1}$. 
Consider the following comparison differential system

$$
\begin{aligned}
\frac{d y(t)}{d t} & =B(0) e^{-\gamma \tau} y(t-\tau) \\
& -\left(\frac{\beta}{1+a M+b M^{2}} \rho+\eta\right) y(t),
\end{aligned}
$$

for $t>n T+\tau, n>k_{1}$.

From (8), we have $B(0) e^{-\gamma \tau}<\frac{\beta}{1+a M+b M^{2}} \rho+\eta$.

According to Lemma 4, we have $\lim _{t \rightarrow \infty} y(t)=0$.

By the comparison theorem, we have $\limsup S(t)<$ $\lim _{t \rightarrow \infty} y(t)=0$. Incorporating into the positivity of $S(t)$, we know that

$$
\lim _{t \rightarrow \infty} S(t)=0
$$

Therefore, for any $\varepsilon_{1}>0$ (sufficiently small), there exists an integer $k_{2}\left(k_{2} T>k_{1} T+\tau\right)$ such that $S(t)<\varepsilon_{1}$ for all $t>k_{2} T$.

Form system (3) and Lemma 5, we have

$$
-d I(t) \leq \frac{d I(t)}{d t} \leq\left(-d+\beta \varepsilon_{1}\right) I(t) .
$$

Then we have $z_{1}(t) \leq I(t) \leq z_{2}(t)$ and $z_{1}(t) \rightarrow \tilde{I}(t)$, $z_{2}(t) \rightarrow \tilde{I}(t)$ as $t \rightarrow \infty$, while $z_{1}(t)$ and $z_{2}(t)$ are the solutions of

$$
\begin{cases}z_{1}^{\prime}(t)=-d z_{1}(t), & t \neq n T, \\ z_{1}\left(t^{+}\right)=z_{1}(t)+\mu, & t=n T, \\ z_{1}\left(0^{+}\right)=I\left(0^{+}\right), & \end{cases}
$$

and

$$
\begin{cases}z_{2}^{\prime}(t)=\left(-d+\beta \varepsilon_{1}\right) z_{2}(t), & t \neq n T, \\ z_{2}\left(t^{+}\right)=z_{2}(t)+\mu, & t=n T, \\ z_{2}\left(0^{+}\right)=I\left(0^{+}\right), & \end{cases}
$$

respectively, $\quad \tilde{z}_{2}(t)=\frac{\mu \exp \left(\left(-d+\beta \varepsilon_{1}\right)(t-n T)\right)}{1-\exp \left(\left(-d+\beta \varepsilon_{1}\right) T\right)}$ for $n T$ $<t \leq(n+1) T$. Therefore, for any $\varepsilon_{2}>0$, there exists an integer $k_{3}, n>k_{3}$ such that

$$
\tilde{I}(t)-\varepsilon_{2}<I(t)<\tilde{z}_{2}(t)+\varepsilon_{2} \text {. for } t>n T .
$$

Let $\varepsilon_{2} \rightarrow 0$, we get $\tilde{z}_{2}(t) \rightarrow \tilde{I}(t)$ Hence $I(t) \rightarrow \tilde{I}(t)$ as $t \rightarrow \infty$. This completes the proof.

\subsection{Permanence}

Persistence (or permanence) is an important property of dynamical systems, in this section, we focus on the per- manence of system (3).

Denote

$$
R_{*} \triangleq \frac{\left(B(0) e^{-\gamma \tau}-\eta\right)\left(1-e^{\left(\beta S^{*}-d\right) T}\right)}{\mu \beta} .
$$

where $S^{*}=\frac{1}{\beta}\left[d+\frac{1}{T} \ln \left(1-\frac{\mu \beta}{B(0) e^{-\gamma \tau}-\eta}\right)\right]>0$.

Theorem 2. Suppose $R_{*}>1$. Then there is a positive constant $q$ such that each positive solution $(S(t), I(t))$ of (3) satisfies $S(t) \geq q$, for sufficiently large $t$.

Proof. Let $(S(t), I(t))$ be the solution of system (3) with initial condition (4). Note that the first equation of (3) can be rewritten as

$$
\begin{aligned}
\frac{d S(t)}{d t} & =e^{-\gamma \tau} B(S(t)) S(t)-\eta S(t)-\frac{\beta S(t) I(t)}{1+a S+b S(t)^{2}} \\
& -e^{-\gamma \tau} \frac{d}{d t} \int_{t-\tau}^{t} B(S(\theta)) S(\theta) d \theta .
\end{aligned}
$$

In the following we define:

$$
W(t)=S(t)+e^{-\gamma \tau} \int_{t-\tau}^{t} B(S(\theta)) S(\theta) d \theta .
$$

Then the derivative of $W(t)$ with respect to the solution of system (3) is governed by

$$
\frac{d W}{d t}=\left(e^{-\gamma \tau} B(S(t))-\eta-\frac{\beta I(t)}{1+a S(t)+b S^{2}(t)}\right) S(t) .
$$

Since $R_{*}>1$, we can choose sufficiently small $S^{*}\left(<\frac{d}{\beta}\right)$ and $\xi$ such that

$$
e^{-\gamma \tau} B\left(S^{*}\right)-\eta-\beta\left(\frac{\mu}{1-e^{\left(\beta S^{*}-d\right) T}}+\xi\right)>0 .
$$

We claim that for any $t_{0}>0$, it is impossible that $S(t)<S^{*}$ for all $t \geq t_{0}$. Suppose that the claim is not valid. Then there is a $t_{0}>0$ such that $S(t)<S^{*}$ for all $t \geq t_{0}$. It follows from the second equation of system (3) that for

$$
\frac{d I(t)}{d t}=\frac{\beta S(t) I(t)}{1+a S(t)+b S^{2}(t)}-d I(t) \leq\left(-d+\beta S^{*}\right) I(t)
$$

Consider the comparison impulsive system for $t \geq t_{0}$,

$$
\begin{cases}z^{\prime}(t)=\left(-d+\beta S^{*}\right) z(t), & t \neq n T, \\ z\left(t^{+}\right)=z(t)+\mu, & t=n T,\end{cases}
$$

According to Lemma 1, we get the unique positive periodic solution of system (13)

$$
\tilde{z}(t)=z^{*} e^{\left(\beta S^{*}-d\right)(t-n T)}, \quad n T<t \leq(n+1) T,
$$

is globally asymptotically stable, where $z^{*}=\frac{\mu}{1-e^{\left(\beta S^{*}-d\right) T}}$. 
By the comparison theorem in impulsive differential equations, we know that for any sufficiently small $\varepsilon>0$, there exists a $t_{1}\left(>t_{0}+\tau\right)$ such that

$$
I(t) \leq z^{*}+\varepsilon \triangleq \sigma,
$$

for all $t>t_{1}$. It follows from (12) that $e^{-\gamma \tau} B\left(S^{*}\right)-\eta-\beta \sigma>0$. Further,

$$
W^{\prime}(t)>\left(e^{-\gamma \tau} B\left(S^{*}\right)-\eta-\beta \sigma\right) S(t) \text { for } t \geq t_{1} .
$$

Set

$$
S_{m}=\min _{t \in\left[t_{1}, t_{1}+\tau\right]} S(t),
$$

We will show that $S(t) \geq S_{m}$. for all $t \geq t_{1}>t_{0}$. Otherwise, there is a $h \geq 0$ such that $S(t) \geq S_{m}$ for $t_{1} \leq t \leq t_{1}+\tau+h, \quad S\left(t_{1}+\tau+h\right)=S_{m}$ and $S^{\prime}\left(t_{1}+\tau+h\right)<0$. Accordingly, from the first equation of (3) and the inequality (14) we yield

$$
\begin{aligned}
S^{\prime}\left(t_{1}+\tau+h\right)= & B\left(S\left(t_{1}+h\right)\right) S\left(t_{1}+h\right) e^{-\gamma \tau} \\
& -\frac{\beta S\left(t_{1}+\tau+h\right) I\left(t_{1}+\tau+h\right)}{1+a S\left(t_{1}+\tau+h\right)+b S^{2}\left(t_{1}+\tau+h\right)} \\
& -\eta S\left(t_{1}+\tau+h\right) \\
& \geq\left(B\left(S^{*}\right) e^{-\gamma \tau}-\beta \sigma-\eta\right) S_{m}>0
\end{aligned}
$$

which leads to a contradiction. Therefore $S(t) \geq S_{m}$ for all $t \geq t_{1}$. As a consequence, (15) leads to

$$
W^{\prime}(t)>\left(B\left(S^{*}\right) e^{-\gamma \tau}-\beta \sigma-\eta\right) S_{m}>0
$$

for $t \geq t_{1}$, which implies that $W(t) \rightarrow \infty$ as $t \rightarrow \infty$. This contradicts $W(t) \leq M\left(1+B(0) \tau e^{-\gamma \tau}\right)$. The claim is proved.

By the claim, we need to consider two cases.

Case 1. $S(t) \geq S^{*}$ for all large $t$.

Case 2. $S(t)$ oscillates about $S^{*}$ for that $t$ is large enough. Define

$$
q=\min \left\{\frac{S^{*}}{2}, q_{1}\right\} .
$$

where $q_{1}=S^{*} e^{-(\beta \sigma+\eta) T}$. We want to show that $S(t) \geq q$ for all large $t$. The conclusion is evident in the first case. For the second case, let $t^{*}>0$ and $v>0$ satisfy

$$
S\left(t^{*}\right)=S\left(t^{*}+v\right)=S^{*} \text { and } S(t)<S^{*},
$$

for all $t \in\left(t^{*}, t^{*}+v\right)$, where $t^{*}$ is sufficiently large such that

$$
I(t) \leq \sigma \text { for } t^{*}<t<t^{*}+v,
$$

$S(t)$ is uniformly continuous. The positive solutions of (3) are ultimately bounded and $S(t)$ is not affected by the impulses. Hence, there is a $g(0<g<\tau$ and $g$ is dependent of the choice of $\left.t^{*}\right)$ such that $S(t)>\frac{S^{*}}{2}$ for $t^{*}<t<t^{*}+g$.

If $v<g$, there is nothing to prove. Let us consider the case $g<v<\tau$. Since $S^{\prime}(t)>-(\beta \sigma+\eta) S(t)$ and $S\left(t^{*}\right)=S^{*}$, hence $S(t) \geq q_{1}$ for $t^{*}+g \leq t \leq t^{*}+\tau$.

If $v>\tau$, it is obvious that $S(t) \geq q$ for $t \in\left[t^{*}, t^{*}+\tau\right]$. Then proceeding exactly as the proof for the above claim, we see that $S(t) \geq q$ for $t \in\left[t^{*}+\tau, t^{*}+v\right]$, because the kind of interval $t \in\left[t^{*}, t^{*}+v\right]$ is chosen in an arbitrary way (we only need $t^{*}$ to be large). We concluded that $S(t) \geq q$ for all large $t$. In the second case, in view of our above discussion, the choice of $q$ is independent of the positive solution, and we proved that any positive solution of (3) satisfies $S(t) \geq q$ for all sufficiently large $t$. This completes the proof.

Theorem 3. Suppose $R_{*}>1$. Then system (1) is permanent.

Proof. Denote $(L(t), S(t), I(t))$ be any solution of system (1). From the second equation of system (3) and Theorem 2, we have

$$
\frac{d I(t)}{d t} \geq I(t)\left(\frac{\beta q}{1+a M+b M^{2}}-d\right) .
$$

Let $\frac{\beta q}{1+a M+b M^{2}} \triangleq A$, it is easy to get $I(t) \rightarrow \infty$ if $A \geq d$, so we can always obtain the positive lower boundary by the theorem of differential equations. Otherwise, by the same argument as those in the proof of Theorem 1, we have $\liminf _{t \rightarrow \infty} I(t) \geq p$, where

$$
p=\frac{\mu e^{(A-d) T}}{1-e^{(A-d) T}}-\varepsilon .
$$

In view of Theorem 2, the first equation of system (1) becomes

$$
\frac{d L(t)}{d t} \geq B(M) q-M B(0) e^{-\gamma \tau}-\gamma L(t) .
$$

It is easy to obtain

$$
\liminf _{t \rightarrow \infty} L(t) \geq \delta
$$

where $\delta=\frac{B(M) q-M B(0) e^{-\gamma \tau}}{\gamma}-\varepsilon$. By Theorem 2 and the above discussion, system (1) is permanent. The proof of Theorem 3 is complete.

\section{Numerical Analysis and Discussion}

We have studied a delayed epidemic model with stagestructure and impulses, theoretically analyze the influ- 
ence of impulsive releasing for the infected pest population, and also obtained that the pest-extinction periodic solution of system (1) is globally attractive if the control variable $R^{*}<1$ given by (7), and the system is permanent with $R_{*}>1$ which is given by (11). We know that, besides the release amount of infectious pests each time, the period of impulsive vaccination and the effective contact rate play an important role in the dynamical behavior of the system. In the following, we will specially analyze the influence of the release amount of infectious pests to the dynamical system. We assume $B(S)=e^{-S}$, and consider the hypothetical set of parameter values as $\gamma=0.45, \quad \tau=1, a=0.1, \quad b=0.01, \quad \beta=0.75, \quad \eta=0.5$, $d=0.6, \quad T=4, \lambda=0.001$.

By Theorem 1 and Theorem 3, we know that when $R^{*}=0.9929<1$, the pest-extinction periodic solution of system (3) is globally attractive and the susceptible pest population becomes extinct (see Figure 1). When the release amount of infected pest reaches a certain value $\mu=0.1$ such that $R_{*}=1.6681>1$, the system (3) is permanent (see Figure 2). So far we have only discussed two cases: $R^{*}<1$ and $R_{*}>1$. But for $R_{*} \leq 1 \leq R^{*}$, the susceptible pest population either becomes extinct (see Figure 3) or coexists to the infect pest population (see Figure 4). According to the above numerical simulation, we think there exists a threshold parameter to decide the extinction of the susceptible pest population and the permanence of the system. These issues will be considered in our future research.

From Figure 5(a), we can observe that: $R_{*}$ is sensitive to $\mu$ value as $\mu$ is small enough, whereas not sensitive to $\mu$ value as $\mu>1.5$. We can also get the similar phenomenon from Figure 5(b). We hope that our results will provide an insight to pest management practicing.

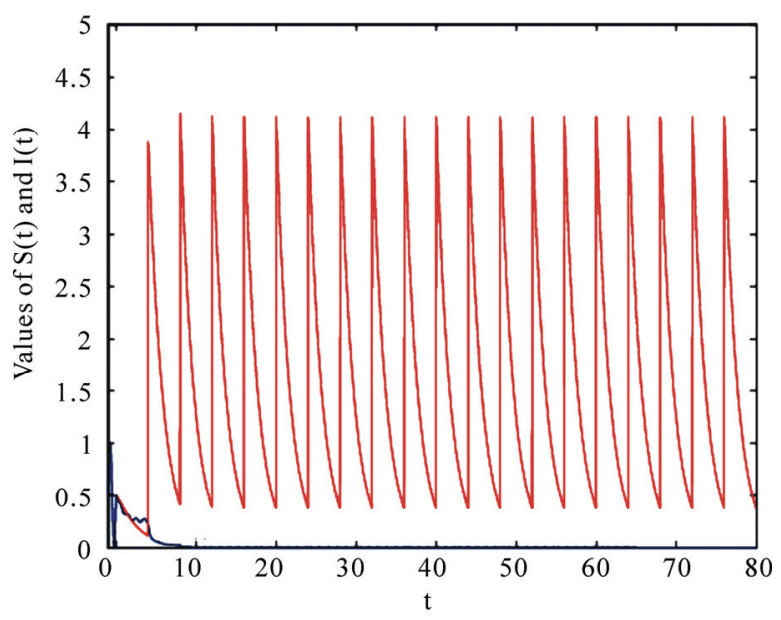

Figure 1. Dynamical behavior of system (3) with $\mu=3.8$, $R^{*}=0.9929<1$.

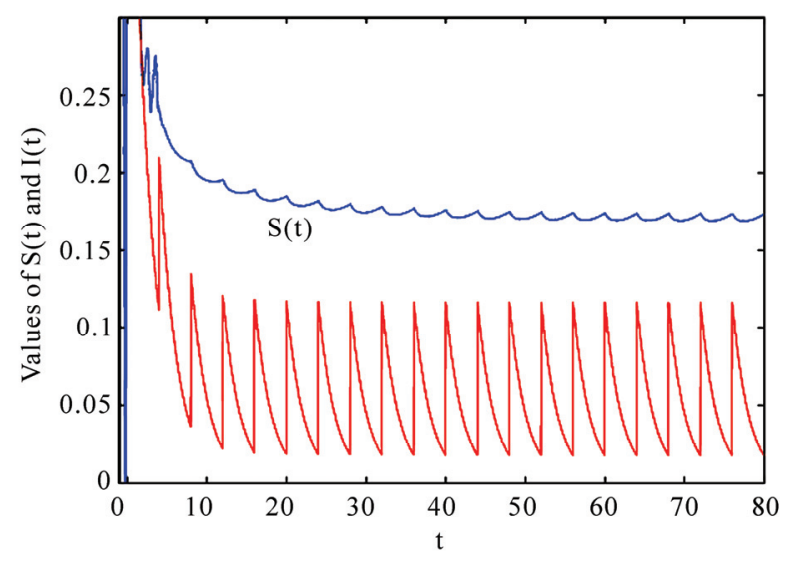

Figure 2. Dynamical behavzior of system (3) with $\mu=0.1$, $R_{*}=1.6681>1$.

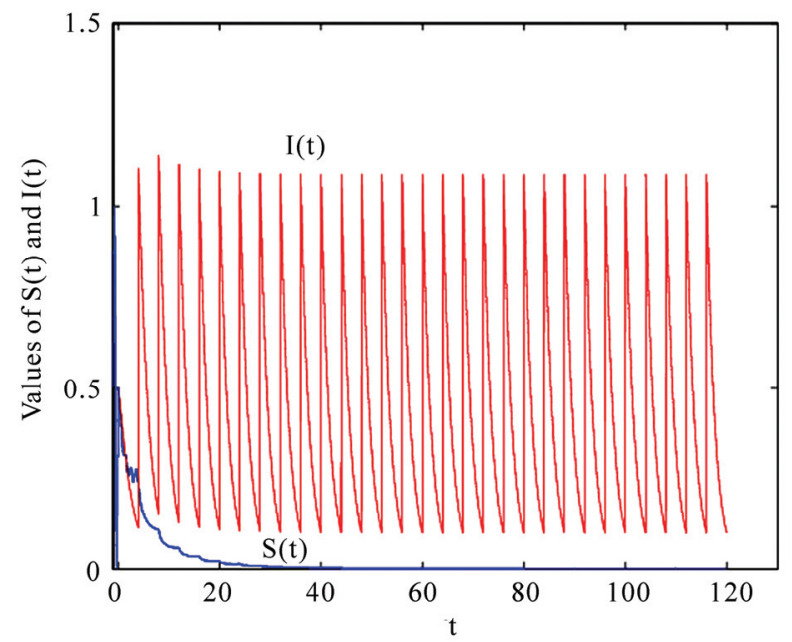

Figure 3. Dynamical behavior of system (3) with $\mu=1$, $R_{*}=0.1669<1$ and $R^{*}=2.2026>1$.

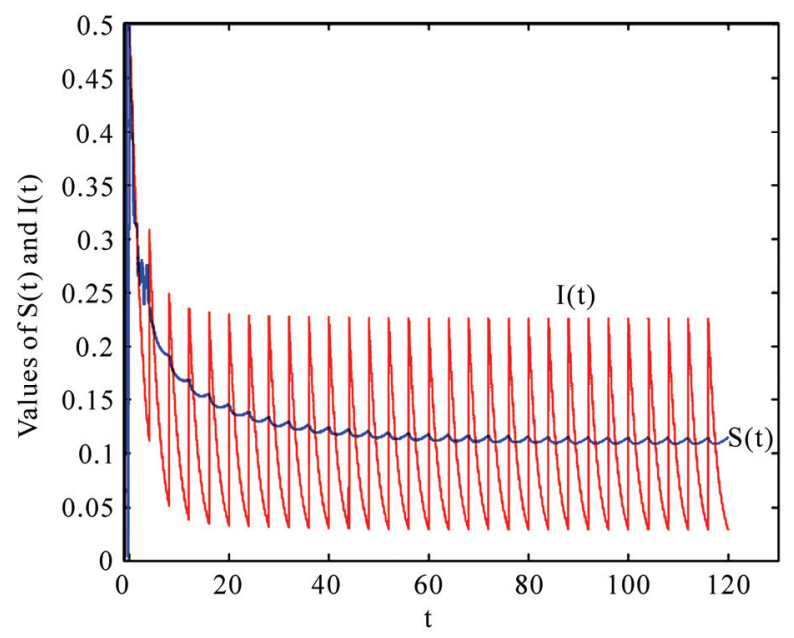

Figure 4. Dynamical behavior of system (3) with $\mu=0.2$, $R_{*}=0.8343<1$ and $R^{*}=9.5212>1$. 


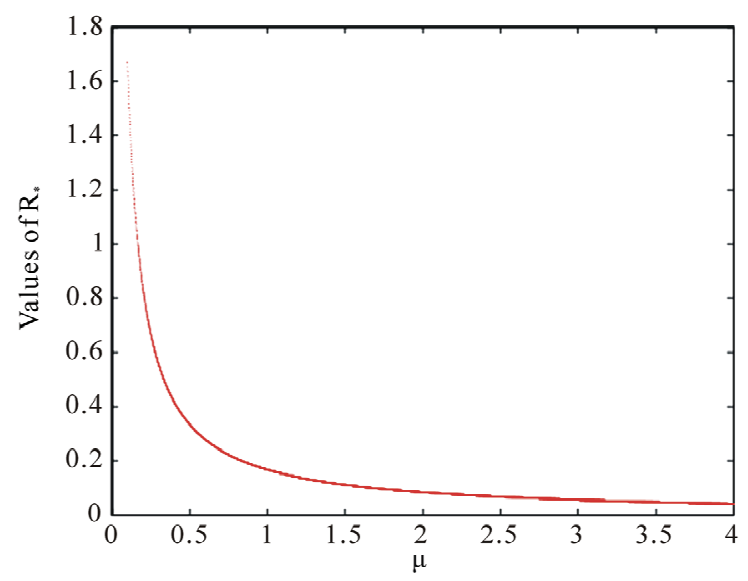

(a)

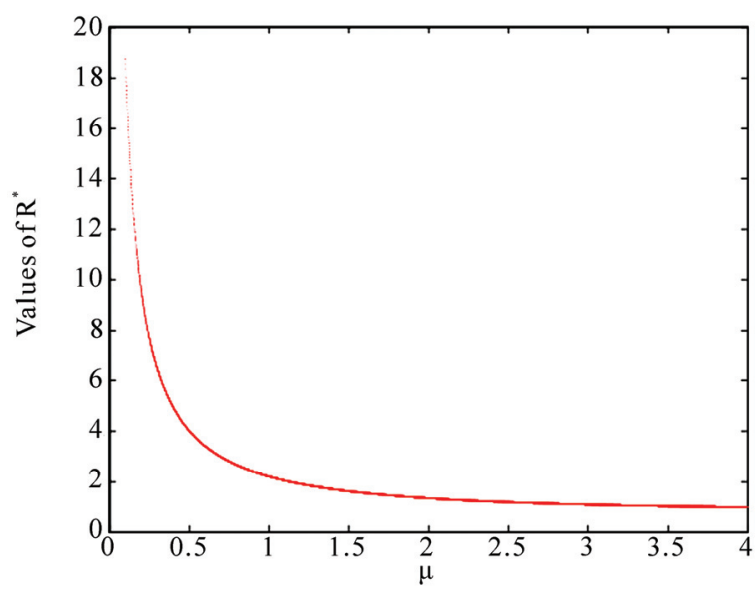

(b)

Figure 5. The sensitive analysis of $\mu$ to $R_{*}$ and $R^{*}$. (a) $\mu-R_{*} ;$ (b) $\mu-R^{*}$.

\section{Acknowledgements}

The research have been supported by The Natural Science Foundation of China (10971037), The National Key Technologies R \& D Program of China (2008BAI68B01), The Postgraduate Innovation Fund of Jiangxi Province (YC09A124).

\section{References}

[1] R. Q. Shi and L. S. Chen, "An Impulsive Predator-Prey Model with Disease in the Prey for Integrated Pest
Management," Communications in Nonlinear Science and Numerical Simulation, Vol. 15, No. 2, 2010, pp. 421429.

[2] J. A. Cui and X. Y. Song, "Permanence of a Predator-Prey System with Stage Structure," Discrete Continuous Dynamical Systems-Series B, Vol. 4, No. 3, 2004, pp. 547554.

[3] Y. N. Xiao and L. S. Chen, "Global Stability of a Predator-Prey System with Stage Structure for the Predator," Acta Mathematica Sinica, Vol. 20, No. 1, 2004, pp. 6370.

[4] H. Zhang, L. S. Chen and J. J. Nieto, "A Delayed Epidemic Model with Stage-Structure and Pulses for Pest Management Strategy," Nonlinear Analysis: Real World Applications, Vol. 9, No. 4, 2008, pp. 1714-1726.

[5] X. Wang, Y. D. Tao and X. Y. Song, "Mathematical Model for the Control of a Pest Population with Impulsive Perturbations on Diseased Pest," Applied Mathematical Modelling, Vol. 33, No. 7, 2009, pp. 3099-3106.

[6] H. W. Hethcote, "The Mathematics of Infectious Disease," Siam Review, Vol. 42, No. 4, 2002, pp. 599-653.

[7] R. M. Anderson, R. M. May and B. Anderson, "Infectious Diseases of Human: Dynamics and Control," Oxford Science Publications, Oxford, 1991.

[8] R. M. Anderson and R. M. May, "Population Biological of Infectious Diseases," Springer Berlin-Heidelberg, New York, 1982.

[9] W. M. Liu, S. A. Levin and Y, Lwasa, "Infuence of Nonlinear Incidence Rates upon the Behavior of SIRS Epidemiological Models," Journal of Mathematical Biology, Vol. 23, No. 2, 1986, pp. 187-204.

[10] T. Lindstrom, "A Generalized Uniqueness Theorem for Limit Cycles in a Predator-Prey System," Acta Academic Akoensis, Series B, Vol. 49, No. 2, 1989, pp. 1-9.

[11] J. J. Jiao, X. Z. Meng ang L. S. Chen, "Global Attractivity and Permanence of a Stage-Structured Pest Management SI Model with Time Delay and Diseased Pest Impulsive Transmission," Chaos, Solitons and Fractals, Vol. 38, No. 3, 2008, pp. 658-668.

[12] V. Lakshmikantham, D. D. Bainov and P. Simeonov, "Theory of Impulsive Differential Equations," World Scientific, Singapor, 1989.

[13] Y. Kuang, "Delay Differential Equation with Application in Population Dynamics," Academic Press, New York, 1993. 